\title{
Prospects for Higgs and SM measurements at the HL-LHC
}

\author{
Alexander A. Savin ${ }^{1,2, a}$ \\ ${ }^{1}$ University of Wisconsin, Madison, USA \\ ${ }^{2}$ on leave from NPI MSU, Moscow, Russia
}

\begin{abstract}
After a succesful startup of the LHC scientific program that has led to discovery of the Higgs boson it is time to make plans for the future. The high luminosity LHC (HLLHC) project is discussed, plans for possible Higgs and SM measurements are reviewed.
\end{abstract}

\section{Introduction}

The HL-LHC project is recently suggested as one of the LHC upgrade steps for 2025. By that time the LHC experiments are expected to collect $300 \mathrm{fb}^{-1}$ of data, where the integrated luminosity is summed over the whole data taking period starting from 2010. The HL-LHC will provide the same amount of integrated luminosity within a year, thus reaching total amount of $3000 \mathrm{fb}^{-1}$ by 2035 . This plan requires an increase of the nominal LHC luminosity by a factor of five to seven, to $5 \times 10^{34}$ $\mathrm{cm}^{-2} \mathrm{~s}^{-1}$ at 140 events (pileup) per bunch crossing that will happen every $25 \mathrm{~ns}$. To better understand the experiment and accelerator requirements, one needs to investigate the physics goals that can be achieved with such amount of data. This presentation covers a few topics based on our current proven knowledge of the standard model (SM). There are many other SUSY and exotica models that are out of the scope of this talk.

\section{Simulation and detector performance}

The studies presented in this article use generator level simulations convoluted with our best knowledge of the expected detector performance. Work on the experiments, reconstruction and physics analyses will continue in parallel, thus the estimates presented at this stage are still preliminary. Both ATLAS and CMS proposals [1,2] are similar in many aspects, since they are based on the same initial physics motivations. Both experiments are planning to include tracking information in the level one trigger systems, thus improving the background rejection at the very first step of the data taking. The calorimeter performance in forward/backward regions is considered to be an important issue, tracking and efficiency of b-tagging in the high pileup scenario were studied in detail by both experiments.

\section{The proposals for the Higgs physics program}

Proposals for Higgs physics program at the HL-LHC are focused on studying the Higgs boson properties with all possible decay channels. First measurements of the Higgs boson couplings are already

\footnotetext{
${ }^{\mathrm{a}}$ On behalf of the ATLAS and CMS collaborations, e-mail: asavin@ mail.cern.ch
} 


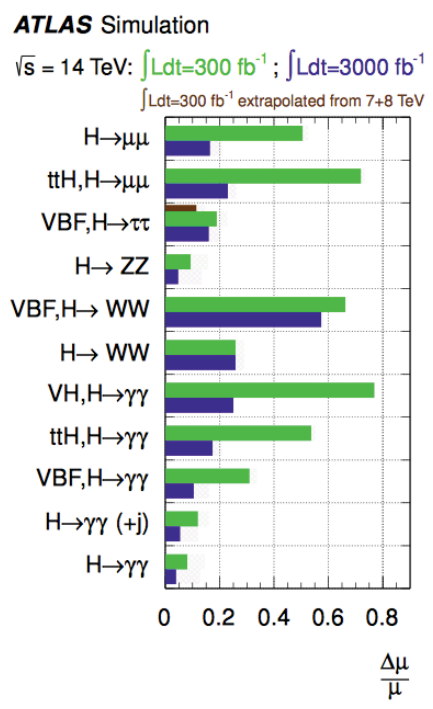

Figure 1. Expected measurements precision on the signal strength $\mu=(\sigma \cdot B R) /(\sigma \cdot B R)_{S M}$ in different Higgs boson decay channels.

started and can be substantially improved at the HL-LHC, where also rare decay channels can be established. Figure 1 shows the estimated measurements precision in different Higgs boson decay channels for the signal strength $\mu$ with respect to the SM Higgs boson expectation for assumed integrated luminosities of $300 \mathrm{fb}^{-1}$ and $3000 \mathrm{fb}^{-1}$. The $\gamma \gamma$ and ZZ channels profit most due to improved statistical and systematic uncertainities. Rare channels, like $\mu \mu$, also gain a lot. Improvement in the WW remains moderate, since it is more systematics limited. Similar results were obtained by the CMS experiment, where the precision in the measurements of the signal strength was found to improve by approximately $30-50 \%$ for the channels, which do not suffer from the low signal yield and by factor of two or more for the rare decay channels.

To demonstrate the effect of the increased luminosity, the di-photon and di-muon masses are shown in Fig. 2 for the $t \bar{t} H, H \rightarrow \gamma \gamma$ (left) and $H \rightarrow \mu \mu$ (right) channels. The signal is clearly seen on the top of background in the first case, and after background subtraction, in the latter one.

Figure 3 shows the four-lepton mass reconstruction in the $H \rightarrow Z Z$ channel from CMS [4] with improved tracker and calorimeter (blue histograms) and after extending tracking coverage in pseudorapidity (red histograms).

A determination of the Higgs self-interaction is crucial for a measurement of the symmetry breaking. Studying of triple and quartic Higgs vertices is therefore a very important goal, which most probably will never be reached without HL-LHC. Two channels sensitive to the trillinear Higgs couplings were studied: $H H \rightarrow b \bar{b} \gamma \gamma$ and $H H \rightarrow b \bar{b} W^{+} W^{-}$. The latter one is identical to $t \bar{t}$-production that gives a large background and therefore no constraints on the Higgs self-coupling can be yet obtained, the work is ongoing. The $H H \rightarrow b \bar{b} \gamma \gamma$ has predicted yield of 260 events in $3000 \mathrm{fb}^{-1}$ and after all selection requirements a signal yield of approximately 11 events is expected, with the irreducible $\gamma \gamma b \bar{b}$ background decreased from 300000 to approximately one event. The overall background yield after all selection requirements is 19 events, mainly from $t \bar{t} H$ process. Thus approximately $3 \sigma$ sensitivity for the double-Higgs production can be achieved by each experiment. In the CMS studies [3] the ef- 

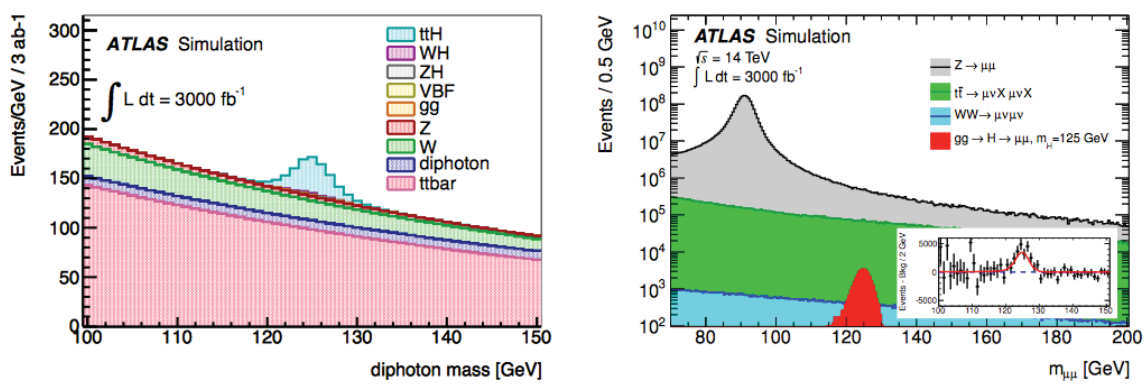

Figure 2. The di-photon and di-muon invariant masses for $t \bar{t} H, H \rightarrow \gamma \gamma$ (left) and $H \rightarrow \mu \mu$ (right) channels. The inset shows the expectation for the $H \rightarrow \mu \mu$ signal after subtraction of the fitted background.

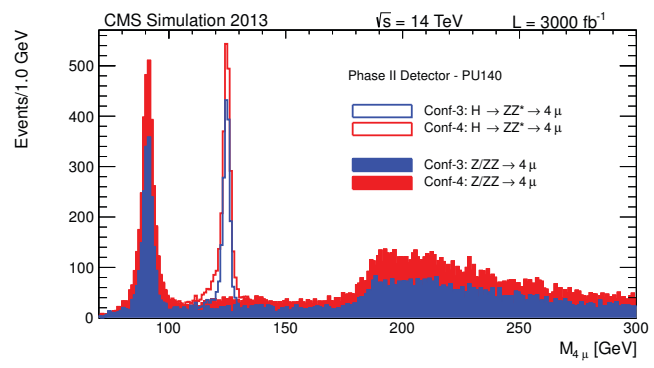

Figure 3. The four-lepton mass reconstructed by CMS in $H \rightarrow Z Z$ channel. Conf.-3 corresponds to improved tracking and calorimeter parts of the detector, Conf.- 4 has in addition an extended tracking to $|\eta|<4$.

fects of the photon selection, b-tagging efficiency and jet energy resolution were studied. It was shown that to achieve the goal of measuring the di-Higgs production cross section to $50 \%$ precision with one experiment and HL-LHC pileup conditions, some improvements on the reconstruction/detector side are still needed.

\section{The proposals for the SM measurements}

The rise of the vector boson scattering (VBS) cross section in the longitudinal mode may lead to violation of unitarity at the scale of $\approx 1 \mathrm{TeV}$. This is why measurement of the VBS energy dependence is very important. The study was done using sensitivity of ATLAS measurements to new high-mass resonances. The $p p \rightarrow Z Z+2 j \rightarrow 4 l+2 j$ channel is used, since it has fully-reconstructible $\mathrm{ZZ}$ resonance peak. The leading jet-jet mass is required to exceed $1 \mathrm{TeV}$, to reduce contribution from non-VBS processes. Figure 4 (left) shows the ZZ mass distribution for data set corresponding to 3000 $\mathrm{fb}^{-1}$ of integrated luminosity and coupling parameter $g=1.75$. In this approach moving from 300 to $3000 \mathrm{fb}^{-1}$ increases the significance of the possible observation in case of background only hypotheses from 1.7 to $5.5 \sigma$.

Similar studies of EWK WZ production were performed by CMS [5]. Figure 4 (right) shows the $\mathrm{WZ}$ transverse mass in WZjj events, where the mass of the jet-jet system is required to exceed $1.2 \mathrm{TeV}$. 

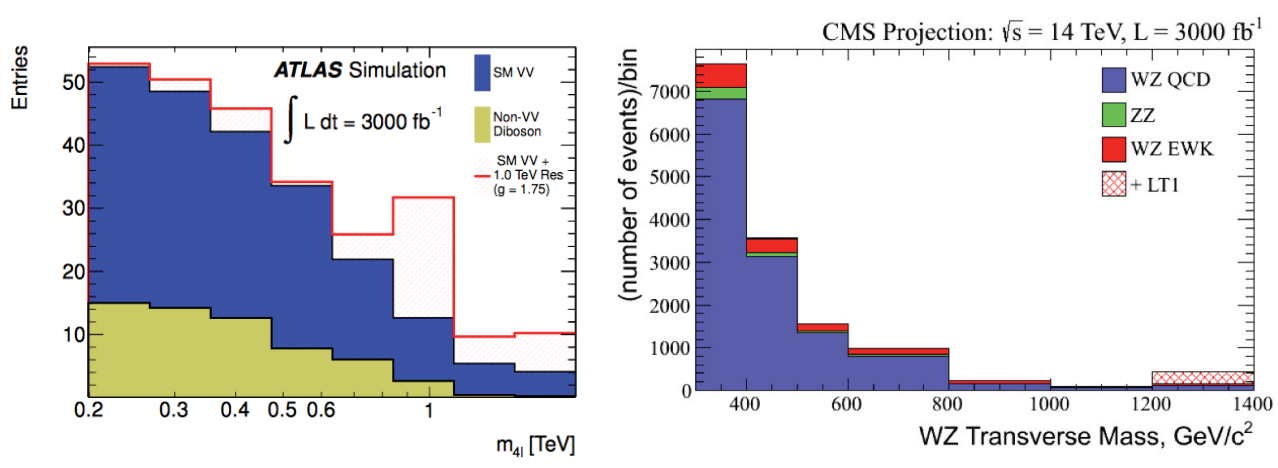

Figure 4. (left) The reconstructed four-lepton mass $\left(m_{4 l}\right)$ spectrum for $p p \rightarrow Z Z+2 j \rightarrow 4 l+2 j$ channel after requiring $m_{j j}>1 \mathrm{TeV}$. (right) The WZ transverse mass. The additional contribution of an aQGC signal is illustrated by the red hatched histogram.

In addition to the SM signal an anomalous quartic gauge coupling (aQGC) contribution was injected, to demonstrate the possible effect at $3000 \mathrm{fb}^{-1}$ of data.

The HL-LHC results may help to significantly improve the experimental and theoretical uncertainties of different measurements. The major uncertainties (approximately 10\%) are due to restricted knowledge of the next-next-to-leading order QCD effects. The effect is estimated by varying the renormalization and factorization scales for the theoretical calculations up and down. These uncertainties can be improved by having more precise inclusive cross section measurements. Selection uncertainties (approximately 10\%) are especially important in the measurements, where vetoing of additional jets is required. These uncertainties can be reduced with precise inclusive and differential cross section measurements. The PDF uncertainties and fundamental constraints (2-7\%) can be also improved exploring high statistics data samples.

\section{Conclusions}

The proposed HL-LHC upgrade offers a unique opportunity to study properties of recently discovered Higgs boson. The first look at the physics outcome of the HL-LHC project demonstrates that an increase in the integrated luminosity to $3000 \mathrm{fb}^{-1}$ will significantly improve our understanding of the $\mathrm{SM}$ and will establish decays and processes, which are not accessible otherwise.

\section{References}

[1] ATLAS Collaboration,CERN-LHCC-2012-022, 2012

[2] CMS Collaboration, arXiv:1307.7135, 2013

[3] CMS Collaboration, CMS-PAS-FTR-13-001, 2013

[4] CMS Collaboration, CMS-PAS-FTR-13-003, 2013

[5] CMS Collaboration, CMS-PAS-FTR-13-006, 2013 